\title{
Promoting HIV indicator condition-guided testing in hospital settings (PROTEST 2.0): study protocol for a multicentre interventional study
}

Saskia J. Bogers ${ }^{1 *}$ (D, Maarten F. Schim van der Loeff ${ }^{1,2}$, Udi Davidovich², Anders Boyd ${ }^{2,3}$, Marc van der Valk', Kees Brinkman ${ }^{4}$, Godelieve J. de Bree ${ }^{1}$, Peter Reiss ${ }^{1,3,5,6}$, Jan E. A. M. van Bergen ${ }^{7,8}$, and Suzanne E. Geerlings ${ }^{1}$, on behalf of the HIV Transmission Elimination AMsterdam (H-TEAM) Consortium

\begin{abstract}
Background: Late presentation remains a key barrier towards controlling the HIV epidemic. Indicator conditions (ICS) are those that are AIDS-defining, associated with a prevalence of undiagnosed HIV $>0.1 \%$, or whose clinical management would be impeded if an HIV infection were undiagnosed. IC-guided HIV testing is an effective strategy in identifying undiagnosed HIV, but opportunities for earlier HIV diagnosis through IC-guided testing are being missed. We present a protocol for an interventional study to improve awareness of IC-guided testing and increase HIV testing in patients presenting with ICs in a hospital setting.

Methods: We designed a multicentre interventional study to be implemented at five hospitals in the region of Amsterdam, the Netherlands. Seven ICs were selected for which HIV test ratios (proportion of patients with an IC tested for HIV) will be measured: tuberculosis, cervical/vulvar cancer or high-grade cervical/vulvar dysplasia, malignant lymphoma, hepatitis B and C, and peripheral neuropathy. Prior to the intervention, a baseline assessment of HIV test ratios across ICS will be performed in eligible patients (IC diagnosed January 2015 through May 2020, $\geq 18$ years, not known HIV positive) and an assessment of barriers and facilitators for HIV testing amongst relevant specialties will be conducted using qualitative (interviews) and quantitative methods (questionnaires). The intervention phase will consist of an educational intervention, including presentation of baseline results as competitive graphical audit and feedback combined with discussion on implementation and opportunities for improvement. The effect of the intervention will be assessed by comparing HIV test ratios of the pre-intervention and post-intervention periods. The primary endpoint is the HIV test ratio within \pm 3 months of IC diagnosis. Secondary endpoints are the HIV test ratio within \pm 6 months of diagnosis, ratio ever tested for HIV, HIV positivity percentage, proportion of late presenters and proportion with known HIV status prior to initiating treatment for their IC.
\end{abstract}

\footnotetext{
* Correspondence: s.j.bogers@amsterdamumc.nl

'Department of Internal Medicine, Division of Infectious Diseases, Amsterdam

University Medical Centers, University of Amsterdam, room D3-226,

Meibergdreef 9, 1105 AZ Amsterdam, the Netherlands

Full list of author information is available at the end of the article
}

\section{$\triangle B M C$}

C C The Author(s). 2021 Open Access This article is licensed under a Creative Commons Attribution 4.0 International License, which permits use, sharing, adaptation, distribution and reproduction in any medium or format, as long as you give appropriate credit to the original author(s) and the source, provide a link to the Creative Commons licence, and indicate if changes were made. The images or other third party material in this article are included in the article's Creative Commons licence, unless indicated otherwise in a credit line to the material. If material is not included in the article's Creative Commons licence and your intended use is not permitted by statutory regulation or exceeds the permitted use, you will need to obtain permission directly from the copyright holder. To view a copy of this licence, visit http://creativecommons.org/licenses/by/4.0/. The Creative Commons Public Domain Dedication waiver (http://creativecommons.org/publicdomain/zero/1.0/) applies to the data made available in this article, unless otherwise stated in a credit line to the data. 
Discussion: This protocol presents a strategy aimed at increasing awareness of the benefits of IC-guided testing and increasing HIV testing in patients presenting with ICs in hospital settings to identify undiagnosed HIV in Amsterdam, the Netherlands.

Trial registration: Dutch trial registry: NL7521. Registered 14 February 2019.

Keywords: Indicator condition, HIV testing, Healthcare quality improvement, Implementation, Multifaceted intervention

\section{Background}

In our efforts to complete the 'last mile' towards ending the HIV epidemic, timely diagnosis of HIV remains a key focal point. Globally, about $19 \%$ of the estimated 38.0 million people living with HIV (PLHIV) were unaware of their HIV status in 2019 [1]. In Europe and Central Asia, one in five PLHIV remain undiagnosed and half of new diagnoses in the European Union are at a late stage of infection (CD4 count $<350$ cells $/ \mathrm{mm}^{3}$ or presenting with an AIDS-defining event) [2]. These figures are worrisome as late presentation is associated with higher morbidity and mortality, poorer response to combination antiretroviral therapy (cART) and onward transmission of HIV [3-6].

One of the strategies to improve timely HIV diagnosis is testing for HIV in all patients diagnosed with an indicator condition (IC). ICs are defined as conditions that are AIDS-defining, that are associated with a prevalence of undiagnosed HIV $>0.1 \%$ (the threshold for costeffectiveness in HIV testing $[7,8])$, or whose clinical management would be adversely affected if HIV infection were not identified. The HIV Indicator Diseases across Europe Study (HIDES) and the subsequent HIDE S II study $[9,10]$ identified various ICs associated with an HIV prevalence of over $0.1 \%$. Currently, over 50 ICs for which HIV testing is recommended are recognised and numerous studies have shown that IC-guided HIV testing is an effective approach to identify undiagnosed PLHIV [11-15]. IC-guided testing also has the advantage that discussing patient risk factors for HIV, which still poses a barrier for some physicians [16, 17], can be bypassed. As a result, HIV testing and care guidelines across Europe have now recommended IC-guided testing [18]. However, various studies have recently shown low adherence to these recommendations. Although they confirmed a prevalence of undiagnosed HIV $>0.1 \%$ amongst patients diagnosed with ICs or, conversely, a high prevalence of ICs amongst newly diagnosed PLHIV, there were consistently low HIV testing ratios in patients presenting with ICs and thus missed opportunities for earlier HIV diagnosis [19-24]. Furthermore, in 2017, the majority of specialty guidelines for ICs did not recommend HIV testing, making awareness amongst medical specialties other than those actively involved in HIV care less likely [25].

In the Netherlands, an estimated 8\% of PLHIV is unaware of their diagnosis and over half of all newly diagnosed cases involve late presentation [26]. In the hospital setting, $69 \%$ of new HIV diagnoses are latestage [26]. Previous research has shown that there have been missed opportunities to identify undiagnosed PLHIV through IC-guided testing [14]. As an estimated $27 \%$ of PLHIV in the Netherlands live in Amsterdam and over one in five HIV diagnoses are made there, a city-based approach to curb the Dutch HIV epidemic is essential. This led to the establishment of the HIV Transmission Elimination Amsterdam (H-TEAM) consortium in 2014. It deploys a city-based combination intervention strategy focussing on all parts of the HIV prevention and care cascade [27]. The H-TEAM designed an interventional study to promote IC-guided HIV testing at hospitals in the region of Amsterdam. Here, we describe the details of a protocol for an interventional study to (1) generate awareness about ICs and the importance of IC-guided HIV testing amongst physicians working in hospitals, and (2) improve the HIV test ratio in ICs amongst different medical specialties in the region of Amsterdam, the Netherlands.

\section{Methods \\ Setting and study design}

We designed a multicentre interventional study that will take place at 5 hospitals (two university hospitals, two non-academic teaching hospitals and one non-teaching hospital) in the region of Amsterdam, the Netherlands.

The development of the study consisted of three phases; an elicitation phase, a design phase, and an implementation phase. The elicitation and design phases have already been completed, while the implementation phase is currently taking place (as of June 2020). During the elicitation phase, a group of four experts, including an infectious diseases physician, a general practitioner, an epidemiologist, and a behavioural scientist, identified essential elements for an empirically based intervention. Additionally, two infectious diseases physicians from two participating hospitals were consulted on the perceived 
feasibility of the identified elements. During the design phase, the study protocol and evaluation plan were composed, with additional consultation from an expert on methodological approaches and statistical analysis. During the implementation phase, we will implement the educational intervention and assess its impact. The preintervention HIV test ratio (i.e. the proportion of patients presenting with an IC who are tested for HIV) will be compared to the post-intervention test ratio for seven selected ICs. To this end, pre-intervention test data over a period of 5.5 years (January 2015 through May 2020) will be retrospectively collected. As all participating hospitals have utilised their current electronic health record (EHR) software from 2015, allowing readily available data, the starting point of the pre-intervention period was selected at that year. The intervention period will last 6 months and its effect will be evaluated for a period of 1 year from the start of the intervention (June 2020 through May 2021, Fig. 1).

\section{Selection of indicator conditions}

Seven ICs were selected for inclusion in this study: tuberculosis (TB), cervical cancer or high-grade cervical dysplasia, vulvar cancer or high-grade vulvar dysplasia, malignant lymphoma, hepatitis B (HBV), hepatitis C (HCV) and peripheral neuropathy (Table 1). These ICs were selected as they are managed by several different medical specialties (pulmonology, gynaecology, haematology, gastroenterology and neurology) and their evidence of being associated with HIV is variable. For example, the association of HIV and TB has been extensively documented, but less evidence on the association between HIV and peripheral neuropathy is available. We additionally selected these ICs based on their relatively high incidence, based on reports by the various specialty associations.

\section{Patient selection and inclusion}

The HIV testing ratio will be assessed using patient data from EHRs. Eligible patients will be identified using national disease billing codes. Patients of $\geq 18$ years, diagnosed with one of the selected ICs will be eligible for inclusion (Fig. 2). The following patients will be excluded: (1) patients with a known HIV infection prior to presenting with the selected IC and (2) patients that are diagnosed and treated for their IC at another hospital, and the relevant billing code was only recorded in the EHR for administrative purposes. Patients who are referred by another physician for a second opinion or transferred from another hospital will be included. ICspecific selection criteria will additionally be applied (Table 1). In both the pre- and post-intervention period, all eligible patients from the first university hospital will be included. For the other four hospitals, data from all eligible patients will be included if there are $\leq 500$ patients per IC; while data from a random sample of 500 patients will be assessed for eligibility and, if eligible, included if the number of patients per IC exceeds 500 . This was done to keep workload manageable as the added precision of inclusions $>500$ is limited. Eligible patients will be given the opportunity to opt-out of the use of their EHR data.

\section{Assessments}

For all included patients, year of birth, sex, socioeconomic status (SES; as derived from the postal code of residence) and whether deceased (including date of death) will be recorded. For women, any pregnancy at the time of IC diagnosis will be recorded. Additionally,

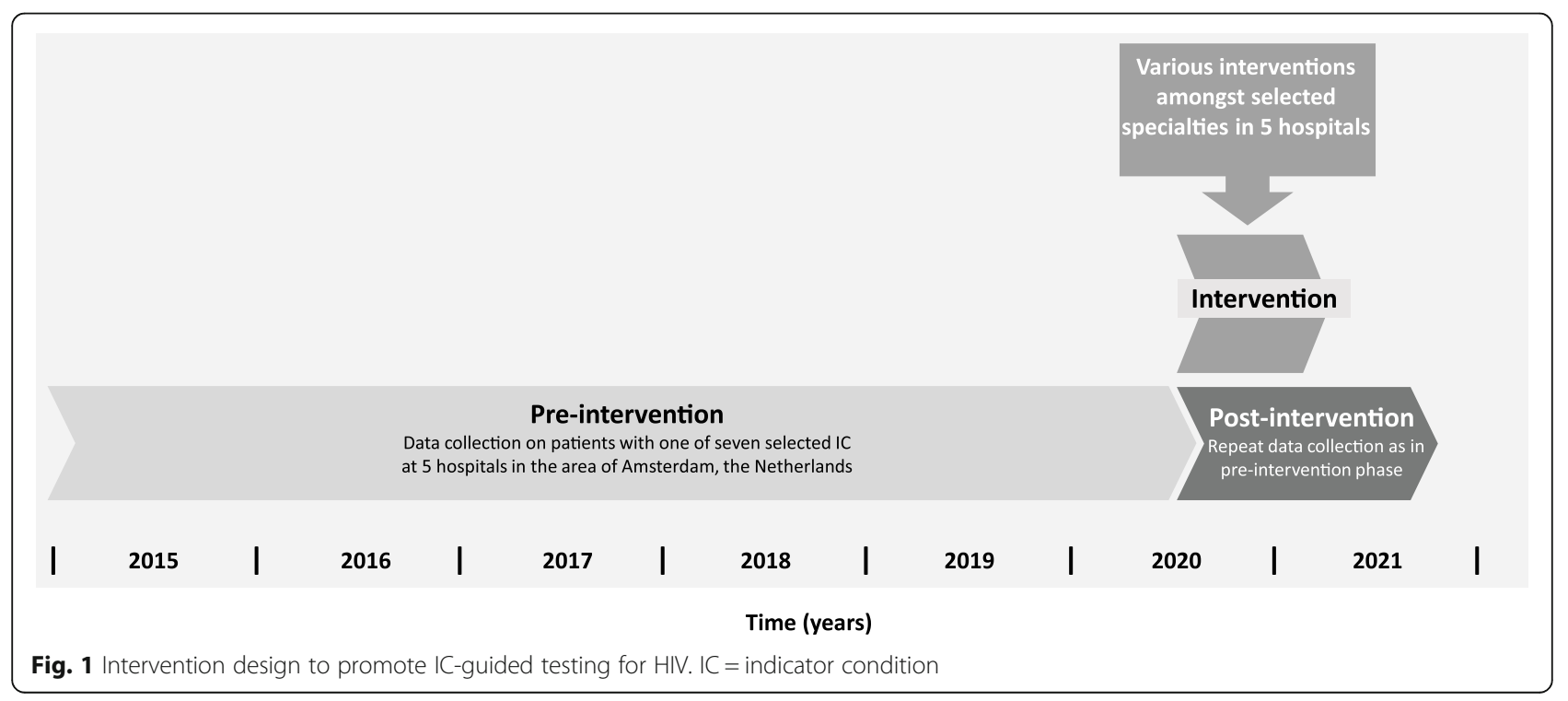


Table 1 Selected indicator conditions, associated specialties, and specific selection criteria for eligibility

\begin{tabular}{|c|c|c|}
\hline Indicator condition & Main Specialty & Indicator condition-specific selection criteria \\
\hline Tuberculosis & Pulmonology & Patients with latent M. tuberculosis infection, but no tuberculosis disease, are excluded \\
\hline $\begin{array}{l}\text { Cervical cancer or CIN } \\
3+\end{array}$ & Gynaecology & $\begin{array}{l}\text { Patients without biopsy-confirmed high-grade dysplasia (CIN 3+) or carcinoma (invasive or non-invasive) } \\
\text { are excluded }\end{array}$ \\
\hline Vulvar cancer or VIN $3+$ & Gynaecology & $\begin{array}{l}\text { Patients without biopsy-confirmed high-grade dysplasia (VIN 3+) or carcinoma (invasive or non-invasive) } \\
\text { are excluded }\end{array}$ \\
\hline Malignant lymphoma & Haematology & $\begin{array}{l}\text { All types of malignant lymphoma, including all subtypes of Hodgkin's lymphoma and non-Hodgkin } \\
\text { lymphoma are included }\end{array}$ \\
\hline Hepatitis B & Gastroenterology & Both acute and chronic hepatitis B cases are included \\
\hline Hepatitis C & Gastroenterology & Both acute and chronic hepatitis C cases are included \\
\hline Peripheral neuropathy & Neurology & $\begin{array}{l}\text { Patients with known diabetes mellitus before presenting and patients for whom no diagnostic laboratory } \\
\text { workup was indicated are excluded }\end{array}$ \\
\hline
\end{tabular}

CIN cervical intraepithelial neoplasia, VIN vulvar intraepithelial neoplasia

the date of diagnosis and, if applicable, treatment of the specific IC will be recorded. To determine outcome measures, if, when and where an HIV test was performed will be recorded. To this end, all laboratory results, scanned documents, patient communication and referral letters in the EHR will be searched. All female patients with an EHR-recorded pregnancy after January 2004 will be assumed to have been tested for HIV during pregnancy, as all pregnant women in the Netherlands are tested for HIV on an opt-out basis, as part of the antenatal care programme, and the number opting out is negligible [28]. When no HIV test was performed during the diagnostic work-up for the IC, the EHR will be searched to assess whether a reason was given by the healthcare provider for not offering an HIV test or by the patient for declining the test. If the result of an HIVtest was positive, we will record the CD4 count at diagnosis. Electronic Case Report Forms (eCRFs) will be used for data collection using Castor (Castor Electronic Data Capture, Amsterdam, the Netherlands). As a quality control check, $10 \%$ of eCRFs per IC and hospital will be randomly selected and verified by a second researcher. If the discrepancy in findings is $>2.5 \%$, all eCRFs for that IC and hospital will be verified by the second researcher.

\section{Endpoints}

The primary endpoint is the HIV test ratio (i.e. the proportion of patients with an IC who were tested for HIV) within \pm 3 months of IC diagnosis ( 3 months before to 3 months after diagnosis). Secondary endpoints are the HIV test ratio within \pm 6 months of IC diagnosis; proportion of patients presenting with an IC that were ever tested for HIV; HIV positivity ratio (i.e. number of positive HIV tests of the total number of HIV tests performed), both for the \pm 3 month and \pm 6 month window; proportion of new HIV diagnoses that are late-stage (defined as having a CD4 count $<350$ cells $/ \mathrm{mm}^{3}$ at diagnosis); and proportion of patients tested for HIV before initiating treatment for their IC.

\section{Intervention strategy}

A multifaceted intervention strategy will be used to improve HIV testing at different medical specialties who primarily diagnose and treat patients with the selected ICs (Table 2). The interventions will be tailored to specialty-specific circumstances. Opportunities for intervention will be identified through qualitative and quantitative research, specifically by addressing barriers for IC-guided testing amongst professionals and their work settings $[29,30]$. First, an overview of IC-guided HIV testing recommendations will be made from the local and national specialty guidelines of the selected ICs. For relevant guidelines without such recommendations, the possibility to amend these guidelines will be explored. Second, all medical specialists and residents from each involved specialty at the participating hospitals will be invited to complete an online questionnaire on barriers and facilitators for HIV testing in patients with ICs related to their discipline. To this end, a questionnaire was developed based on the Attitude-Social norm-self Efficacy (ASE) model [31, 32], which is an evidence-based instrument to assess behavior and its determinants in healthcare personnel (Supplementary appendix 1). The questionnaire will be distributed via email by contact persons representing each specialty at each hospital. The proportion responding will be calculated as the number of respondents divided by the number of recipients. Third, attitudes towards IC-guided HIV testing, and opportunities for improvement that fit the respondent's specialty and hospital, will be assessed through semi-structured interviews with medical specialists and residents. Individuals who respond to the online questionnaire and are willing to provide contact information will be recruited for these interviews. The contact persons of the various specialties will also be invited to participate in the interviews. The outcomes and 


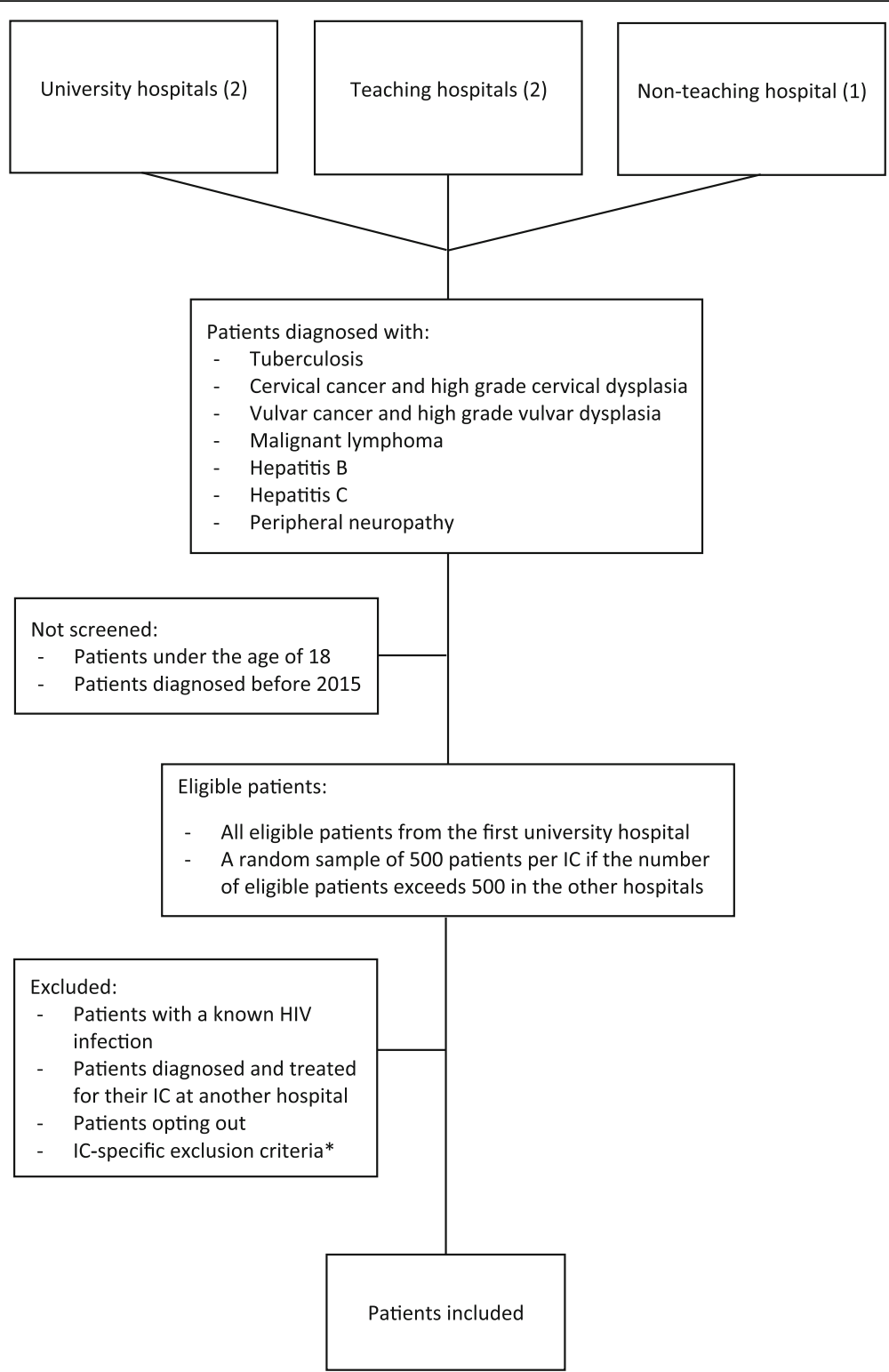

Fig. 2 Flowchart of patient inclusion. * See Table 1 for an overview. IC = indicator condition

opportunities for improvement from these interviews will be used for the educational intervention meetings (Supplementary appendix 2).

We will offer to host an educational meeting of about 30-45 min for each medical specialty at each participating hospital. During these educational meetings, the current state of the HIV epidemic in the Netherlands, evidence on the relation between HIV and the relevant ICs and evidence on IC-guided testing for HIV will first be presented. In addition to the ICs selected for this study, the entire list of currently recognised ICs will be presented with the aim of highlighting other ICs relevant to the specialty and bringing a more comprehensive awareness of IC-guided testing for HIV. Baseline HIV test ratios for all hospitals will be presented. This technique is known as competitive graphical audit and feedback, which was chosen because of its effectiveness in improving guideline adherence [33]. Finally, the results of the questionnaires and interviews, and identified barriers and opportunities for improvement, will be presented, followed by an interactive discussion on opportunities to improve HIV testing. When suggestions for improvement strategies are made by the participants during this discussion or other phases of the implementation period, we will offer assistance in implementation. At the end of the meeting, educational materials (pocket cards and posters) will be handed out to remind participants of the topics discussed. Participants will be 
Table 2 Planned study components to promote indicator condition-guided testing for HIV

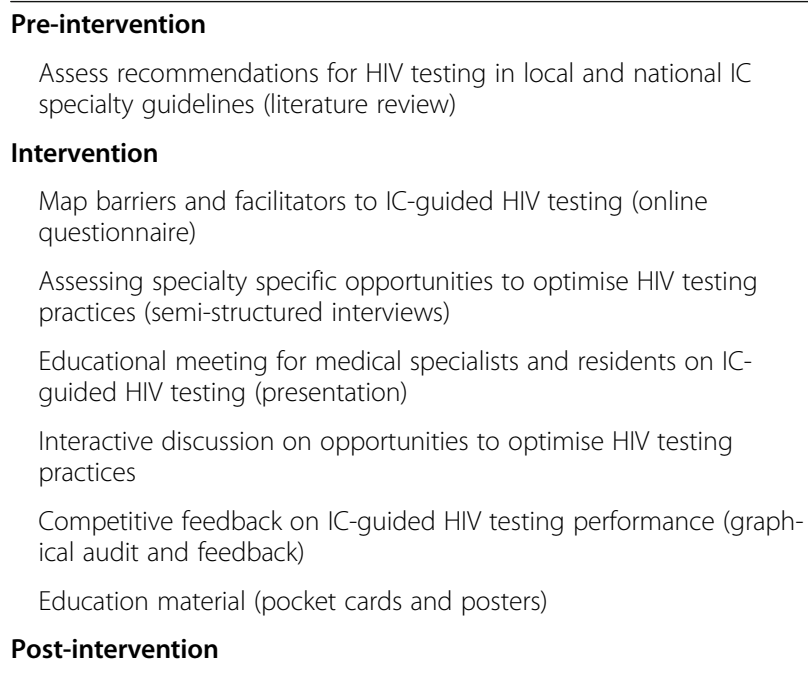

Educational meeting for medical specialists and residents on ICguided HIV testing (presentation)

Interactive discussion on opportunities to optimise HIV testing practices

Competitive feedback on IC-guided HIV testing performance (graphical audit and feedback)

Education material (pocket cards and posters)

\section{Post-intervention}

Reporting of post-intervention feedback on IC-guided HIV testing performance to participating specialties (graphical audit and feedback)

IC indicator condition

informed that the effect of the intervention will be assessed through a post-intervention assessment of the HIV test ratio and that these results will be reported back to all participating hospitals.

\section{Statistical analysis}

The number of patients with ICs will be reported per IC, per hospital, and per period (in the pre- or postintervention periods) as well as the number and percentage of patients with an IC who were tested for HIV within \pm 3 months of IC diagnosis. Additionally, the number and percentage of patients with an IC who were tested for HIV within \pm 6 months of IC diagnosis and the number and percentage of patients with an IC who were ever tested for HIV will be reported.

A time-series approach using segmented, Poisson regression will be used to evaluate the effect of the intervention. We will first model the HIV test ratio as a function of calendar time (in quarter-year periods), intervention period (pre- versus post-intervention), and the interaction between the two. If the interaction term in the model is significant (i.e. differences in slopes), the effect of the intervention will be determined by testing the parameter estimate of the interaction term. If the interaction term is non-significant (i.e. no difference in slopes), the interaction term of the model above will be removed and the effect of the intervention will be tested by the intervention term. Assuming no difference in slopes, average recruitment rate of 31 patients/IC per quarter, an increase in testing from 60 to $80 \%$ for four ICs (TB, HBV, HCV, malignant lymphoma) and from 12 to $30 \%$ for the three remaining ICs (cervical cancer/ high-grade dysplasia, vulvar cancer/high-grade dysplasia, peripheral neuropathy) in the pre- versus postintervention periods, respectively (unpublished data), there will be $>95 \%$ power to determine a difference between intervention arms based on a simulation of 2000 runs. Potential confounding variables will also be added to the regression model. $P$-values will be obtained using a Wald $X^{2}$ test and a $p$-value of $<0.05$ will be considered statistically significant. Subgroup analyses will be performed for each IC and hospital separately, provided that there is $\geq 1$ individual tested during each intervention period within that stratum. Analyses will be performed using Stata (v15.1, StataCorp, USA).

\section{Discussion}

We describe the design of an interventional study that aims to generate awareness amongst hospital-based physicians of the importance of testing for HIV in patients presenting with ICs and to increase the proportion of patients with an IC who are tested for HIV in various medical specialties.

The designed interventional study has several strengths. During the elicitation phase, we identified multiple intervention strategies based on qualitative and quantitative research that can be used simultaneously. This allows us to implement various innovations in healthcare that have been proven successful in other contexts $[29,30]$. Likewise, implementing graphical audit and feedback into the educational intervention for this study will hopefully bring about an effective strategy to increase awareness of ICguided testing for physicians [33, 34].

During the design phase, we selected a wide array of ICs, some of which have been thoroughly established as indicator conditions and already have included HIV testing as part of their specialty guidelines (e.g. TB, HCV), while for others, this has not been the case (e.g. high grade cervical dysplasia and peripheral neuropathy). Additionally, we considered that specialists from the infectious diseases department will have already been attune to HIV testing. Selecting ICs likely to be diagnosed at a broad range of other departments will ensure an intervention that has a much wider reach and thus has increased generalizability.

For the implementation phase, we will use a timeframe of 6 months around diagnosis of an IC (i.e. 3 months before and 3 months after) when calculating the primary endpoint. One previous study used a period of 3 months after IC diagnosis [35], which might inevitably exclude HIV tests performed during the workup leading to an IC diagnosis. Other studies have used 1 month or 6 months as part of their timeframe [13, 36, 37]. Since testing for HIV is not always the first priority after diagnosing an IC, 1 month may be too restrictive. Conversely, allowing up 
to 6 months between the diagnosis of an IC and an HIV test may be too long to prevent adverse outcomes related to undiagnosed HIV infection, especially when the patient is a late presenter of HIV infection. Nevertheless, we will use the latter cut-off as part of a secondary analysis.

One limitation that will undoubtedly arise when evaluating the effect of our intervention is the lack of control group, as no data from departments or hospitals that are unexposed to the interventions will be collected. However, the Poisson regression model used in this study will include a time component to establish any changes in HIV testing from the moment our interventions are applied. Second, because the study uses data from EHRs, certain patient characteristics, such as ethnicity and sexual risk behaviour, cannot be included, as they are not consistently reported by physicians. Consequently, no adjustment for these possible confounding variables can be made. However, as international guidelines recommend testing for HIV in all patients presenting with an IC, regardless of other patient risk factors, these are considered inessential for this study. Finally, as we will evaluate the effect of our educational intervention by comparing the HIV test ratio over a period of 1 year from the start of the intervention to a baseline assessment, we will be unable to assess whether any effect would be sustainable in the long term.

In conclusion, we have developed a protocol for an empirically based interventional study to create awareness of and improve IC-guided testing in a hospital setting. During the implementation phase, analysis comparing HIV testing before and after its implementation will determine whether this approach is effective in improving IC-guided testing for HIV at hospitals located in the Amsterdam region, with the aim of facilitating earlier identification of PLHIV who currently remain undiagnosed.

\section{Abbreviations}

ASE: Attitude - Social norm - self Efficacy; CART: Combination Antiretroviral Therapy; CIN: Cervical Intraepithelial Neoplasia; eCRF: Electronic Case Report Forms; EHR: Electronic Health Record; HBV: Hepatitis B; HCV: Hepatitis C; HIDE S: HIV Indicator Diseases across Europe Study; H-TEAM: HIV Transmission Elimination Amsterdam consortium; IC: Indicator Condition; PLHIV: People living with HIV; SES: Socio-Economic Status; TB: Tuberculosis; VIN: Vulvar Intraepithelial Neoplasia

\section{Supplementary Information}

The online version contains supplementary material available at https://doi. org/10.1186/s12879-021-06183-8.

Additional file 1: Supplementary appendix 1. Online questionnaire. This supplementary appendix provides additional information on the development, distribution and content of the online questionnaire that will be used in this study.

Additional file 2: Supplementary appendix 2. Semi-structured interview guide. This supplementary appendix provides additional information on the recruitment and enrolment for the semi-structured interviews, as well as the interview guide that will be used in this study.

\section{Acknowledgements}

We gratefully acknowledge all site investigators of the participating hospitals; Dr. Kim Sigaloff, Dr. Judith Branger and Dr. Nejma Bokhizzou. We thank Karlijn Groen for her preparatory work in this project.

The authors thank the H-TEAM Steering Committee: H-TEAM Steering Committee: J.E.A.M. van Bergen ${ }^{1 ; 2 ; 3}$, P. Brokx ${ }^{4}$, F. Deug ${ }^{1}$, M. Heidenrijk ${ }^{5}$, M. Prins ${ }^{6 ;}$, P. Reiss ${ }^{5 ; 8}$ (chair), M. van der Valk ${ }^{7}$, the H-TEAM Core Project Group: J.E.A.M. van Bergen ${ }^{1 ; 2 ; 3}$, G.J. de Bree ${ }^{5 ; 7}$ (chair), P. Brokx ${ }^{5}$, U. Davidovich ${ }^{6}$, S.E. Geerlings ${ }^{7}$, E. Hoornenborg ${ }^{6}$, A. Oomen ${ }^{1}$, A. van Sighem ${ }^{8}$, W. Zuilhof ${ }^{1}$, the H-TEAM Project Management: N. Schat ${ }^{5}$, and all H-TEAM additional collaborators: R.C.A. Achterbergh $^{6}$, M. van Agtmael ${ }^{9}$, J. Ananworanich ${ }^{10}$, D. Van de Beek ${ }^{11}$, G.E.L. van den Berk ${ }^{12}$, D. Bezemer ${ }^{8}$, A. van Bijnen ${ }^{1}$, W.L. Blok ${ }^{12}$, S. Bogers ${ }^{7}$, M. Bomers $^{9}$, C.A.B. Boucher ${ }^{13}$, W. Brokking ${ }^{14}$, D. Burger ${ }^{15}$, K. Brinkman $^{12}$, N. Brinkman $^{16}$, M. de Bruin ${ }^{17}$, S. Bruisten ${ }^{6}$, L. Coyer ${ }^{6}$, R. van Crevel $^{18}$, C.G. Daans ${ }^{6 ; 19}$, L. Dellemann ${ }^{1}$, M. Dijkstra ${ }^{6}$, Y.T. van Duijnhoven ${ }^{6}, A . v^{2}$ an Eeden ${ }^{14}$, L. Elsenburg ${ }^{14}$, M.A.M. van den Elshout ${ }^{6}$, C. Ester $^{8}$, E. Ersan ${ }^{2}$, P. E.V. Felipa ${ }^{2}$, P.H.J. Frissen ${ }^{12}$, T.B.H. Geijtenbeek ${ }^{20}$, M.H. Godfried', J. van Gool', A. Goorhuis ${ }^{7}$, M. Groot ${ }^{14}$, C.A. Hankins ${ }^{5}$, A. Heijnen ${ }^{21 ; 22}$, M.M.J Hillebregt ${ }^{8}$, A. Hogewoning ${ }^{6}$, M. Hommenga ${ }^{6}$, J.W. Hovius', Y. Janssen ${ }^{16}$, K. de Jong ${ }^{6}$, V. Jongen ${ }^{6}$, N.A. Kootstra ${ }^{23}$, R.A. Koup ${ }^{24}$, F.P. Kroon ${ }^{25}$, T.J.W. van de Laar ${ }^{26 ; 27}$, F. Lauw ${ }^{28}$, M. M. van Leeuwen $^{6}$, K. Lettinga ${ }^{29}$, I. Linde ${ }^{6}$, D.S.E. Loomans ${ }^{6}$, J.T. van der Meer ${ }^{7}$, T. Mouhebati ${ }^{1}$, B.J. Mulder ${ }^{6}$, J. Mulder ${ }^{30}$, F.J. Nellen ${ }^{7}$, A. Nijsters ${ }^{1}$, H. Nobel ${ }^{7}$, P.

Oostvogel ${ }^{6}$, E.L.M. Op de Coul ${ }^{3}$, E. Peters ${ }^{9}$, I.S. Peters ${ }^{6}$, T. van der Poll ${ }^{7}$, O. Ratmann $^{31}$, C. Rokx ${ }^{32}$, M.S. van Rooijen ${ }^{6}$, M.F. Schim van der Loeff6;33, W.E.M.

Schoute $^{12}$, G.J. Sonder ${ }^{6}, J$. Veenstra ${ }^{29}$, A. Verbon ${ }^{32}$, F. Verdult ${ }^{4}$, J. de Vocht ${ }^{9}$, H.J. de Vries ${ }^{6 ; 33 ; 34}$, S. Vrouenraets ${ }^{29}$, M. van Vugt ${ }^{7}$, W.J. Wiersinga ${ }^{7}$, F.W. Wit ${ }^{7}{ }^{7}$, L.R. Woittiez ${ }^{7}$, S. Zaheri ${ }^{8}$, P. Zantkuijl ${ }^{1}$, M.C. van Zelm ${ }^{35}$, A. Żakowicz ${ }^{36}$, H.M.L. Zimmermann 6 .

1 Soa Aids Nederland, Amsterdam, the Netherlands.

2 Department of General Practice, Amsterdam UMC - location AMC,

University of Amsterdam, Amsterdam, the Netherlands.

3 Epidemiology and Surveillance Unit, Center for Infectious Disease Control, National Institute of Public Health and the Environment, the Netherlands.

${ }^{4}$ Dutch Association of PLHIV, Amsterdam, the Netherlands.

${ }^{5}$ Department of Global Health, Amsterdam UMC - location AMC, and

Amsterdam Institute for Global Health and Development, Amsterdam, the Netherlands.

${ }^{6}$ Department of Infectious Diseases, Public Health Service of Amsterdam, Amsterdam, the Netherlands.

${ }^{7}$ Department of Internal Medicine, Division of Infectious Diseases,

Amsterdam UMC - location AMC, Amsterdam, the Netherlands.

${ }^{8}$ Stichting HIV Monitoring, Amsterdam, the Netherlands.

${ }^{9}$ Department of Internal Medicine, Amsterdam UMC - location VUMC,

Amsterdam, the Netherlands.

${ }^{10}$ US Military HIV Research Program and the Henry M. Jackson Foundation for the Advancement of Military Medicine, Bethesda, United States.

${ }^{11}$ Center of Infection and Immunity Amsterdam (CINIMA), Department of Neurology, Amsterdam UMC - location AMC, Amsterdam, the Netherlands.

${ }^{12}$ Department of internal medicine, OLVG - location East, Amsterdam, the Netherlands.

${ }^{13}$ Department of viro-science, Erasmus Medical Center Rotterdam, Rotterdam, the Netherlands.

${ }^{14}$ DC Klinieken, Amsterdam, the Netherlands.

${ }^{15}$ Department of Pharmacy, Radboud University Nijmegen Medical Center, Nijmegen, the Netherlands.

${ }^{16}$ Primary Care Amsterdam and Almere (Elaa), Amsterdam, the Netherlands. ${ }^{17}$ Aberdeen Health Psychology Group, Institute of Applied Health Sciences, University of Aberdeen, Aberdeen, United Kingdom.

18 Department of Internal Medicine, Radboud University Nijmegen Medical Center, Nijmegen, the Netherlands.

${ }^{19}$ Center of Expertise on Gender Dysphoria, Amsterdam UMC - location

VUMC, Amsterdam, the Netherlands.

${ }^{20}$ Laboratory of Experimental Immunology, Amsterdam UMC - location

AMC Amsterdam, the Netherlands.

${ }^{21}$ Sexology Center Amsterdam, Amsterdam, the Netherlands.

${ }^{22}$ GP practice Heijnen \& de Meij, Amsterdam, the Netherlands.

${ }^{23}$ Laboratory for Viral Immune Pathogenesis, Amsterdam UMC - location AMC Amsterdam, the Netherlands.

${ }^{24}$ Immunology Laboratory, Vaccine Research Center, National Institute of Allergy and Infectious Diseases, National Institutes of Health, Rockville, Maryland, USA. 
25 Department of Infectious Diseases, Leiden University Medical Center, Leiden, the Netherlands.

${ }^{26}$ Department of Medical Microbiology, OLVG, Amsterdam, the Netherlands.

${ }^{27}$ Department of Donor Medicine Research, Laboratory of Blood-borne Infections, Sanquin Research, Amsterdam, the Netherlands.

${ }^{28}$ Department of Internal Medicine, Medical Center Jan van Goyen,

Amsterdam, the Netherlands.

29 Department of Internal Medicine, OLVG - location West, Amsterdam, the Netherlands.

${ }^{30}$ Department of Internal Medicine, Slotervaart Hospital (former), Amsterdam, the Netherlands.

${ }^{31}$ School of Public Health, Faculty of Medicine, Imperial College London, London, United Kingdom.

${ }^{32}$ Department of Internal Medicine and Infectious Diseases, Erasmus Medical Center, Rotterdam, the Netherlands.

${ }^{33}$ Center for Infection and Immunology, Amsterdam (CINIMA), Amsterdam

UMC - location AMC, University of Amsterdam, Amsterdam, the Netherlands.

${ }^{34}$ Department of Dermatology, Amsterdam UMC - location AMC, University

of Amsterdam, Amsterdam, the Netherlands.

35 Department of Virology, Erasmus Medical Center, Rotterdam, the

Netherlands.

${ }^{36}$ AlDS Healthcare Foundation, Amsterdam, the Netherlands.

\section{Authors' contributions}

$\mathrm{SB}, \mathrm{MSL}, \mathrm{UD}, \mathrm{MV}, \mathrm{KB}, \mathrm{JB}$ and $\mathrm{SG}$ designed the study. SB designed the intervention materials. SG arranged funding. SB, MSL, AB and SG outlined the statistical analysis. SB, MSL and SG drafted the manuscript. All authors contributed to, read and approved this final manuscript.

\section{Funding}

PROTest 2.0 is funded by Aids Fonds (grant number: P- 42702) and the $\mathrm{H}$ TEAM. The H-TEAM initiative is supported by Aids Fonds (grant number: 2013169), Stichting Amsterdam Dinner Foundation, Bristol-Myers Squibb International Corp. (study number: Al424-541), Gilead Sciences Europe Ltd. (grant number: PA-HIV-PREP-16-0024), Gilead Sciences (protocol numbers: CO-NL-276-4222, CO-US-276-1712), Janssen Pharmaceutica (reference number: PHNL/JAN/0714/0005b/1912fde), M.A.C AIDS Fund, ViiV Healthcare (PO numbers: 3000268822, 3000747780) and ZonMw (grant number: 522002003). The funders of this study did not play any role in the design of the study and collection, analysis, and interpretation of data or in the writing of this manuscript or the decision to publish.

\section{Availability of data and materials}

Not applicable.

\section{Declarations}

\section{Ethics approval and consent to participate}

The Medical Ethics Committee of the Amsterdam University Medical Centers, University of Amsterdam (Amsterdam UMC-AMC) determined that this study does not meet the definition of medical research involving human subjects as specified in Dutch law. Local feasibility was assessed and established by the Board of Directors in all study hospitals. All patients are given the opportunity to object to the consultation of their medical records and the use of their data for the purpose of this study through an opt-out procedure. All physicians participating in the questionnaires and interviews are asked for their content to participate in this study.

\section{Consent for publication}

Not applicable.

\section{Competing interests}

$A B$ is a member of the editorial board of BMC infectious diseases. All other authors declare that they have no competing interests directly related to this study.

\section{Author details}

'Department of Internal Medicine, Division of Infectious Diseases, Amsterdam University Medical Centers, University of Amsterdam, room D3-226, Meibergdreef 9, 1105 AZ Amsterdam, the Netherlands. ${ }^{2}$ Department of Infectious Diseases, Public Health Service of Amsterdam, Amsterdam, the
Netherlands. ${ }^{3}$ HIV Monitoring Foundation, Amsterdam, the Netherlands. ${ }^{4}$ Department of Internal Medicine, Onze Lieve Vrouwe Gasthuis, Amsterdam, the Netherlands. ${ }^{5}$ Amsterdam Institute for Global Health and Development, Amsterdam, the Netherlands. ${ }^{6}$ Department of Global Health, Amsterdam University Medical Centers, University of Amsterdam, Amsterdam, the Netherlands. ${ }^{7}$ Department of General Practice, Amsterdam University Medical Centers, location Academic Medical Center, University of Amsterdam, Amsterdam, the Netherlands. ${ }^{8}$ STI AIDS Netherlands, Amsterdam, the Netherlands.

Received: 10 December 2020 Accepted: 14 May 2021 Published online: 02 June 2021

\section{References}

1. UNAIDS. Global HIV \& AIDS statistics - 2020 fact sheet. 2020. https://www. unaids.org/en/resources/fact-sheet. Accessed 1 Sept 2020.

2. Brown $A E$, Hayes R, Noori T, et al. HIV in Europe and Central Asia: progress in 2018 towards meeting the UNAIDS 90-90-90 targets. Euro Surveill. 2018; 23(48):1800622.

3. Krentz HB, Auld MC, Gill MJ. The high cost of medical care for patients who present late (CD4 <200 cells/microL) with HIV infection. HIV Med. 2004;5(2): 93-8. https://doi.org/10.1111/j.1468-1293.2004.00193.x.

4. Sabin CA, Smith CJ, Gumley H, Murphy G, Lampe FC, Phillips AN, et al. Late presenters in the era of highly active antiretroviral therapy: uptake of and responses to antiretroviral therapy. AIDS. 2004;18(16):2145-51. https://doi. org/10.1097/00002030-200411050-00006.

5. Cohen MS, Chen YQ, McCauley M, Gamble T, Hosseinipour MC, Kumarasamy N, et al. Prevention of HIV-1 infection with early antiretroviral therapy. N Engl J Med. 2011;365(6):493-505. https://doi.org/10.1056/ NEJMoa1105243.

6. Mocroft A, Lundgren JD, Sabin ML, Monforte A'A, Brockmeyer N, Casabona $J$, et al. Risk factors and outcomes for late presentation for HIV-positive persons in Europe: results from the collaboration of observational HIV epidemiological research Europe study (COHERE). PLoS Med. 2013;10(9): e1001510. https://doi.org/10.1371/journal.pmed.1001510.

7. Bert F, Gualano MR, Biancone P, Brescia V, Camussi E, Martorana M, et al. Cost-effectiveness of HIV screening in high-income countries: a systematic review. Health Policy. 2018;122(5):533-47. https://doi.org/10.1016/j.hea Ithpol.2018.03.007.

8. Yazdanpanah Y, Sloan CE, Charlois-Ou C, le Vu S, Semaille C, Costagliola D, et al. Routine HIV screening in France: clinical impact and cost-effectiveness. PLoS One. 2010;5(10):e13132. https://doi.org/10.1371/journal.pone.0013132.

9. Sullivan AK, Raben D, Reekie J, Rayment M, Mocroft A, Esser S, et al. Feasibility and effectiveness of indicator condition-guided testing for HIV: results from HIDES I (HIV indicator diseases across Europe study). PLoS One. 2013;8(1):e52845. https://doi.org/10.1371/journal.pone.0052845.

10. Raben D, Mocroft A, Rayment M, Mitsura VM, Hadziosmanovic V, Sthoeger ZM, et al. Auditing HIV testing rates across Europe: results from the HIDES 2 study. PLoS One. 2015;10(11):e0140845. https://doi.org/10.1371/journal.pone. 0140845.

11. Menacho I, Sequeira E, Muns M, et al. Comparison of two HIV testing strategies in primary care centres: indicator-condition-guided testing vs. testing of those with non-indicator conditions. HIV Med. 2013;14 Suppl 3:33-7.

12. Omland LH, Legarth R, Ahlstrom MG, Sorensen $H T$, Obel N. Five-year risk of HIV diagnosis subsequent to 147 hospital-based indicator diseases: a Danish nationwide population-based cohort study. Clin Epidemiol. 2016;8:333-40. https://doi.org/10.2147/CLEP.S101288.

13. Scognamiglio P, Chiaradia G, De Carli G, et al. The potential impact of routine testing of individuals with HIV indicator diseases in order to prevent late HIV diagnosis. BMC Infect Dis. 2013;13(1):473. https://doi.org/10.1186/14 71-2334-13-473

14. Joore IK, Arts DL, Kruijer MJ, et al. HIV indicator condition-guided testing to reduce the number of undiagnosed patients and prevent late presentation in a high-prevalence area: a case-control study in primary care. Sex Transm Infect. 2015;91(7):467-72. https://doi.org/10.1136/sextrans-2015-052073.

15. Damery S, Nichols L, Holder R, Ryan R, Wilson S, Warmington S, et al. Assessing the predictive value of HIV indicator conditions in general practice: a case-control study using the THIN database. Br J Gen Pract. 2013; 63(611):e370-7. https://doi.org/10.3399/bjgp13X668159. 
16. Bagchi AD, Davis T. Clinician barriers and facilitators to routine HIV testing: a systematic review of the literature. J Int Assoc Provid AIDS Care. 2020;19: 2325958220936014.

17. Deblonde J, De Koker P, Hamers FF, Fontaine J, Luchters S, Temmerman M. Barriers to HIV testing in Europe: a systematic review. Eur J Pub Health. 2010;20(4):422-32. https://doi.org/10.1093/eurpub/ckp231.

18. HIV Indicator Conditions: Guidance for implementing HIV testing in adults in health care settings. Copenhagen, Denmark: HIV in Europe; 2014. http:// www.eurotest.org/Finalised-Projects/Guidance-HIV-Indicator-Conditions. Accessed 12 Oct 2020

19. Lin YD, Garner SE, Lau JSY, Korman TM, Woolley IJ. Prevalence of HIV indicator conditions in late presenting patients with HIV: a missed opportunity for diagnosis? QJM. 2019;112(1):17-21. https://doi.org/10.1093/ gjmed/hcy223.

20. Levy I, Maor Y, Mahroum N, Olmer L, Wieder A, Litchevski V, et al. Missed opportunities for earlier diagnosis of HIV in patients who presented with advanced HIV disease: a retrospective cohort study. BMJ Open. 2016;6(11): e012721. https://doi.org/10.1136/bmjopen-2016-012721.

21. Agusti C, Montoliu A, Mascort J, et al. Missed opportunities for HIV testing of patients diagnosed with an indicator condition in primary care in Catalonia, Spain. Sex Transm Infect. 2016;92(5):387-92. https://doi.org/10.113 6/sextrans-2015-052328.

22. Bull L, Rayment M. HIV-indicator-condition-driven HIV testing: clinically effective but still rarely implemented. Clin Med (Lond). 2016;16(2):175-9. https://doi.org/10.7861/clinmedicine.16-2-175.

23. Ruutel K, Lemsalu L, Latt S, Opt TH. Monitoring HIV-indicator condition guided HIV testing in Estonia. HIV Med. 2018;19 Suppl 1:47-51.

24. Youssef E, Sanghera T, Bexley A, Hayes M, Perry N, Dosekun O. HIV testing in patients presenting with indicator conditions in outpatient settings: offer and uptake rates, and educational and active interventions. Int J STD AIDS 2018;29(13):1289-94. https://doi.org/10.1177/0956462418781681.

25. Lord E, Stockdale AJ, Malek R, Rae C, Sperle I, Raben D, et al. Evaluation of HIV testing recommendations in specialty guidelines for the management of HIV indicator conditions. HIV Med. 2017;18(4):300-4. https://doi.org/1 $0.1111 /$ hiv. 12430 .

26. van Sighem Al, Wit FWNM, Boyd A, Smit C, Matser A, Reiss P. Monitoring Report 2019. Human immunodeficiency virus (HIV) infection in the Netherlands. Amsterdam: Stichting HIV Monitoring; 2018. https://www.hivmonitoring.nl/nl/resources/monitoring-report-2019. Accessed 12 Oct 2020

27. de Bree GJ, van Sighem A, Zuilhof W, van Bergen J, Prins M, Heidenrijk M, et al. Is reaching 90-90-90 enough to end AIDS? Lessons from Amsterdam. Curr Opin HIV AIDS. 2019;14(6):455-63. https://doi.org/10.1097/COH. 0000000000000586.

28. Rijksinstituut voor Volksgezondheid en Milieu (RIVM). Prenatale Screening, Infectieziekten en Erytrocytenimmunisatie (PSIE) procesmonitor 2017 (Dutch). 2019. https://www.pns.nl/documenten/proces-monitor-psie. Accessed 12-10-2020.

29. Fleuren MA, Paulussen TG, Van Dommelen P, Van Buuren S. Towards a measurement instrument for determinants of innovations. Int J Qual Health Care. 2014;26(5):501-10. https://doi.org/10.1093/intqhc/mzu060.

30. Flottorp SA, Oxman AD, Krause J, Musila NR, Wensing M, Godycki-Cwirko M, et al. A checklist for identifying determinants of practice: a systematic review and synthesis of frameworks and taxonomies of factors that prevent or enable improvements in healthcare professional practice. Implement Sci. 2013:8(1):35. https://doi.org/10.1186/1748-5908-8-35.

31. de Vries H, Dijkstra M, Kuhlman P. Self-efficacy: the third factor besides attitude and subjective norm as a predictor of behavioural intentions. Health Educ Res. 1988;3(3):273-82. https://doi.org/10.1093/her/3.3.273.

32. Schellart AJM, Steenbeek R, Mulders HPG, Anema JR, Kroneman H, Besseling JJM. Can self-reported disability assessment behaviour of insurance physicians be explained? Applying the ASE model. BMC Public Health. 2011; 11(1):576. https://doi.org/10.1186/1471-2458-11-576.

33. Spoorenberg V, Hulscher ME, Geskus RB, et al. A cluster-randomized trial of two strategies to improve antibiotic use for patients with a complicated urinary tract infection. PLoS One. 2015;10(12):e0142672. https://doi.org/10.13 71/journal.pone.0142672.

34. Ivers NM, Grimshaw JM, Jamtvedt G, Flottorp S, O'Brien MA, French SD, et al. Growing literature, stagnant science? Systematic review, metaregression and cumulative analysis of audit and feedback interventions in health care. J Gen Intern Med. 2014;29(11):1534-41. https://doi.org/10.1007/ s11606-014-2913-y.
35. Twisk DE, Joore IK, van Bergen J. Improving early HIV diagnosis, treatment and care in Europe with healthcare provider specific HIV indicator condition list and audits. Sex Transm Infect. 2017;93(8):582. https://doi.org/10.1136/ sextrans-2016-053091.

36. Wohlgemut J, Lawes T, Laing RB. Trends in missed presentations and late HIV diagnosis in a UK teaching hospital: a retrospective comparative cohort study. BMC Infect Dis. 2012;12(1):72. https://doi.org/10.1186/1471-2334-12-72.

37. Gullon A, Verdejo J, de Miguel R, Gomez A, Sanz J. Factors associated with late diagnosis of HIV infection and missed opportunities for earlier testing. AIDS Care. 2016;28(10):1296-300. https://doi.org/10.1080/0954 0121.2016 .1178700

\section{Publisher's Note}

Springer Nature remains neutral with regard to jurisdictional claims in published maps and institutional affiliations.
Ready to submit your research? Choose BMC and benefit from:

- fast, convenient online submission

- thorough peer review by experienced researchers in your field

- rapid publication on acceptance

- support for research data, including large and complex data types

- gold Open Access which fosters wider collaboration and increased citations

- maximum visibility for your research: over $100 \mathrm{M}$ website views per year

At $\mathrm{BMC}$, research is always in progress.

Learn more biomedcentral.com/submissions 